\title{
Vibration absorption with a nonlinear absorder including time delay
}

\author{
S. Bellizzi ${ }^{1}$, K.W. Chung ${ }^{2, a}$, and K. $\mathrm{Hu}^{2,3}$ \\ 1 LMA, CNRS, UPR 7051, Centrale Marseille, Aix-Marseille Univ \\ F-13420 Marseille Cedex 20, France. \\ 2 Department of Mathematics, City University of Hong Kong \\ Tat Chee Avenue, Kowloon, Hong Kong. \\ 3 School of Mathematics and Computational Science, Sun Yat-sen University, Guangzhou, P. R. China.
}

Abstract. In this paper, forced responses are investigated in a one degree-of-freedom linear system subjected to periodic excitation and coupled to a nonlinear energy sink including nonlinear damping with time delay.

\section{Introduction}

A passive control of sound can be achieved using a vibroacoustic coupling between the acoustic field (the primary system) and a geometrically nonlinear thin baffled structure (the strongly nonlinear absorber)[1,2]. The principle is based on the phenomenon called Targeted Energy Transfer (TET) or Energy Pumping [3,4]. If the nonlinear absorber is properly designed for the primary system and its operational conditions, an irreversible energy transfer from the linear system toward the absorber occurs, the energy is dissipated within the absorber damper and the forced dynamic response of the primary system is limited [5]. This means that the nonlinear system behaves like a "sink" where there is motion localization and energy dissipation. In literature, this is also called Nonlinear Energy Sink (NES). The complex dynamics of this kind of coupled systems can be described in terms of resonance capture [4] or nonlinear normal modes [6].

Under periodic external excitation applied to the primary system, the nonlinear absorbers can efficiently reduce the resonance peak by entering the whole system in a quasi-periodic motion with repetitive TET phase[5]. Weakly quasiperiodic responses and strongly quasiperiodic responses (also named "strongly modulated response") can exists or coexist[7]. The very important point is that this peak reduction can occur in a wide frequency band, with the NES adapting itself to the resonance frequencies of the primary system. On the other hand, the NES can operate efficiently only in a limited range of the amplitude. Following these results, design and optimization of the nonlinear absorber have been addressed in $[8,9]$.

The present work aims to analyze the efficiency of a NES including nonlinear damping with time delay as an nonlinear absorber. A complexification method is employed for the investigation.

\section{Description of the model}

The system under consideration is shown in Fig. 1. It consists of a linear oscillator which is the primary system $(M$,

\footnotetext{
a e-mail: makchung@cityu.edu.hk
}

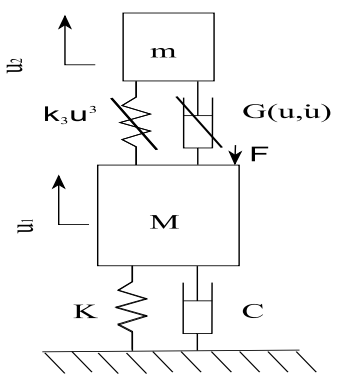

Fig. 1. Schema of the system.

$C$ and $K)$ and an attached nonlinear absorber also named Nonlinear Energy Sink (NES). The NES is composed of a small mass $m$ (relative to $M$ ) attached to the linear substructure via an essential nonlinear spring (cubic) and an active damper with nonlinear damping characteristics defined as

$$
G(u(s-T), \dot{u}(s))=\left\{\begin{array}{l}
c_{m}|\dot{u}(s)| \dot{u}(s),|u(s-T)|<e \\
c_{M}|\dot{u}(s)| \dot{u}(s),|u(s-T)|>e
\end{array}\right.
$$

where $c_{m}$ and $c_{M}$ are two positive constants, $T>0$ defines the time delay and $e$ defines the displacement amplitude threshold for changing the damping characteristics. Note that when $T=0$ and $c_{m}=c_{M}, G$ defines a quadratic damping model. On the other hand, when $T=0$ and $c_{m} \neq c_{M}, G$ defines a piecewise-quadratic damping model. These cases have been considered in [9]. Finally when $T>0, G$ defines a delay piecewise-quadratic damping model.

\subsection{Nondimensional equations of motion}

Using the time normalization

$$
t=\omega_{1}^{\mathrm{p}} s \text { with } \omega_{1}^{\mathrm{p}^{2}}=\frac{K}{M}
$$

combined with re-scaled quantities

$$
x_{1}(t)=u_{1}\left(\frac{t}{\omega_{1}^{\mathrm{p}}}\right) / A, x_{2}(t)=u_{2}\left(\frac{t}{\omega_{1}^{\mathrm{p}}}\right) / A,
$$


the equations of motion can be written in the following nondimensional form

$$
\begin{aligned}
& \ddot{x}_{1}(t)+2 \epsilon \mu \dot{x}_{1}(t)+x_{1}(t)+\epsilon \lambda\left(x_{1}(t)-x_{2}(t)\right)^{3} \\
& \quad+\epsilon g\left(x_{1}(t-\tau)-x_{2}(t-\tau), \dot{x}_{1}(t)-\dot{x}_{2}(t)\right)=\epsilon f(t), \\
& \epsilon \ddot{x}_{2}(t)-\epsilon \lambda\left(x_{1}(t)-x_{2}(t)\right)^{3} \\
& \quad-\epsilon g\left(x_{1}(t-\tau)-x_{2}(t-\tau), \dot{x}_{1}(t)-\dot{x}_{2}(t)\right)=0
\end{aligned}
$$

with

$$
g(x(t-\tau), \dot{x}(t))=\left\{\begin{array}{l}
\lambda_{m}|\dot{x}(t)| \dot{x}(t),|x(t-\tau)|<a \\
\lambda_{M}|\dot{x}(t)| \dot{x}(t),|x(t-\tau)|>a
\end{array},\right.
$$

where $\epsilon=\frac{m}{M}$ is the mass ratio, $\tau=\omega_{1}^{\mathrm{p}} T$ the time delay with respect to the time $t$ and the other parameters $\left(\mu, \lambda, \lambda_{m}, \lambda_{M}\right.$ and $\left.a\right)$ can be easily deduced from the physical parameters.

Assuming that the parameter $\epsilon$ is small $(m<<M)$, it establishes the order of magnitude for the coupling terms, the damping and the excitation force.

The objective now is to characterize the responses of Eqs. (3-4) when the excitation force (applied to the primary system) is of the form

$$
f(t)=A \cos ((1+\epsilon \sigma) t) .
$$

\subsection{Final model}

With the final coordinate transformation

$$
\left\{\begin{array}{l}
x_{1}+\epsilon x_{2}=u \\
x_{1}-x_{2}=w
\end{array},\right.
$$

Eqs. (3-5) are expressed in the following form:

$$
\begin{gathered}
\ddot{u}+2 \epsilon \mu \frac{\dot{u}+\epsilon \dot{w}}{1+\epsilon}+\frac{u+\epsilon w}{1+\epsilon}=\epsilon f(t), \\
\ddot{w}+2 \epsilon \mu \frac{\dot{u}+\epsilon \dot{w}}{1+\epsilon}+\frac{u+\epsilon w}{1+\epsilon}+(1+\epsilon) \lambda w^{3} \\
+(1+\epsilon) g\left(w_{\tau}, \dot{w}\right)=\epsilon f(t)
\end{gathered}
$$

where

$$
g\left(w_{\tau}, \dot{w}\right)=\left\{\begin{array}{l}
\lambda_{m}|\dot{w}| \dot{w},\left|w_{\tau}\right|<a \\
\lambda_{M}|\dot{w}| \dot{w},\left|w_{\tau}\right|>a
\end{array}\right.
$$

To simplify the notation, the time dependence has been omitted in the left hand sides of the equations and the following notation has been used for the delayed term $w_{\tau}=$ $w(t-\tau)$.

As in [9], periodic, quasi-periodic (due to Hopf bifurctaion) and strongly quasiperiodic responses have to be analyzed.

\section{Complexification method}

This section is devoted to the study of periodic regimes when the frequency of excitation is near the resonance frequency of the system.

\subsection{Complexified system}

The complexification method combined to the averaging method will be applied starting from the equations of motion (7-8)

The following complex change of variables is considered

$$
\dot{u}+j u=\varphi_{u} \mathrm{e}^{j t}, \dot{w}+j w=\varphi_{w} \mathrm{e}^{j t}\left(j^{2}=-1\right)
$$

giving

$$
\begin{aligned}
& u=-\frac{j}{2}\left(\varphi_{u} \mathrm{e}^{j t}-\overline{\varphi_{u}} \mathrm{e}^{-j t}\right), \dot{u}=\frac{1}{2}\left(\varphi_{u} \mathrm{e}^{j t}+\overline{\varphi_{u}} \mathrm{e}^{-j t}\right) \\
& \ddot{u}=\left(\dot{\varphi}_{u} \mathrm{e}^{j t}+j \varphi_{u} \mathrm{e}^{j t}\right)-\frac{j}{2}\left(\varphi_{u} \mathrm{e}^{j t}+\overline{\varphi_{u}} \mathrm{e}^{-j t}\right)
\end{aligned}
$$

and similar expressions for $w, \dot{w}$ and $\ddot{w}$ where $\left(^{-}\right)$denotes complex conjugate.

The complex variables $\varphi_{u}$ and $\varphi_{w}$ are assumed as slowly evolving compared to the excitation frequency.

The delayed variable $w_{\tau}$ takes the form

$$
w_{\tau}=-\frac{j}{2}\left(\varphi_{w_{\tau}} \mathrm{e}^{j(t-\tau)}-\overline{\varphi_{w_{\tau}}} \mathrm{e}^{-j(t-\tau)}\right)
$$

which reduces to

$$
w_{\tau}=-\frac{j}{2}\left(\varphi_{w} \mathrm{e}^{j(t-\tau)}-\overline{\varphi_{w}} \mathrm{e}^{-j(t-\tau)}\right)
$$

due to slowly evolution of $\varphi_{w}$. Note that $\left|w_{\tau}\right| \leq\left|\varphi_{w}\right|$.

Substituting Eqs. (11-12) and (14) into Eqs. (7-8) and performing an averaging over one period of the resonance frequency of the system yield the following system of slow modulation

$$
\begin{aligned}
\dot{\varphi}_{u}+j \epsilon & \frac{\left(\varphi_{u}-\varphi_{w}\right)}{2(1+\epsilon)}+\epsilon \mu \frac{\left(\varphi_{u}+\epsilon \varphi_{w}\right)}{1+\epsilon}=\frac{1}{2} \epsilon A \mathrm{e}^{j(\epsilon \sigma t+\phi)} \\
\dot{\varphi}_{w}+j \frac{\left(\varphi_{w}-\varphi_{u}\right)}{2(1+\epsilon)}+\epsilon \mu \frac{\left(\varphi_{u}+\epsilon \varphi_{w}\right)}{1+\epsilon} & \\
+(1+\epsilon) G_{1}+\lambda(1+\epsilon) G_{3} & =\frac{1}{2} \epsilon A \mathrm{e}^{j(\epsilon \sigma t+\phi)}
\end{aligned}
$$

where

$$
G_{3}=\frac{1}{2 \pi} \int_{0}^{2 \pi}(u)^{3} \mathrm{e}^{-j t} d t=-j \frac{3}{8} \varphi_{w}\left|\varphi_{w}\right|^{2}
$$

and

$$
\begin{aligned}
G_{1} & =\frac{1}{2 \pi} \int_{0}^{2 \pi} g\left(w_{\tau}, \dot{w}\right) \mathrm{e}^{-j t} d t \\
& =\varphi_{w}\left|\varphi_{w}\right|\left(G_{1}^{R}\left(\tau,\left|\varphi_{w_{\tau}}\right|\right)+j G_{1}^{I}\left(\tau,\left|\varphi_{w_{\tau}}\right|\right)\right)
\end{aligned}
$$

with $G_{1}^{R}\left(\tau,\left|\varphi_{w}\right|\right)$ and $G_{1}^{I}\left(\tau,\left|\varphi_{w}\right|\right)$ given by

$$
G_{1}^{R}\left(\tau,\left|\varphi_{w}\right|\right)=\frac{4}{3 \pi} \lambda_{m} \text { and } G_{1}^{I}\left(\tau,\left|\varphi_{w}\right|\right)=0
$$

for $\left|\varphi_{w}\right|<a$ and, for $\left|\varphi_{w}\right|>a$, two cases depending on $\tau$ and $t^{*}$ (where $t^{*}$ is defined as $\left|\varphi_{w}\right| \sin t^{*}=a$ with $0 \leq t^{*} \leq \frac{\pi}{2}$ ) are introduced:

Case 1: $\left(0 \leq t^{*}+\tau \leq \frac{\pi}{2}\right.$ and $\left.-\frac{\pi}{2} \leq \tau-t^{*} \leq \frac{\pi}{2}\right)$

$$
\begin{aligned}
G_{1}^{R}\left(\tau,\left|\varphi_{w}\right|\right) & =\frac{1}{6 \pi}\left(8 \lambda_{M}+\left(\lambda_{m}-\lambda_{M}\right) \frac{a}{\left|\varphi_{w}\right|}(9 \cos (\tau)\right. \\
& \left.\left.+\left(3-4 \frac{a^{2}}{\left|\varphi_{w}\right|^{2}}\right) \cos (3 \tau)\right)\right)
\end{aligned}
$$




$$
\begin{aligned}
G_{1}^{I}\left(\tau,\left|\varphi_{w}\right|\right) & =\frac{1}{3 \pi}\left(\lambda_{m}-\lambda_{M}\right) \frac{a}{\left|\varphi_{w}\right|}\left(-3+2 \frac{a^{2}}{\left|\varphi_{w}\right|^{2}}\right. \\
& \left.+\left(-3+4 \frac{a^{2}}{\left|\varphi_{w}\right|^{2}}\right) \cos (2 \tau)\right) \sin (\tau) .
\end{aligned}
$$

Case 2: $\left(\frac{\pi}{2} \leq t^{*}+\tau \leq \pi\right.$ and $\left.-\frac{\pi}{2} \leq \tau-t^{*} \leq \frac{\pi}{2}\right)$

$$
\begin{aligned}
& G_{1}^{R}\left(\tau,\left|\varphi_{w}\right|\right)=\frac{1}{6 \pi}\left(8 \lambda_{m}-\left(\lambda_{m}-\lambda_{M}\right) \sqrt{1-\frac{a^{2}}{\left|\varphi_{w}\right|^{2}}}\right. \\
& \left.\times\left(9 \sin (\tau)+\sin (3 \tau)\left(-4 \frac{a^{2}}{\left|\varphi_{w}\right|^{2}}+1\right)\right)\right) \\
& G_{1}^{I}\left(\tau,\left|\varphi_{w}\right|\right)=-\frac{1}{3 \pi} \cos (\tau)\left(\lambda_{m}-\lambda_{M}\right) \sqrt{1-\frac{a^{2}}{\left|\varphi_{w}\right|^{2}}} \\
& \quad \times\left(2 \frac{a^{2}}{\left|\varphi_{w}\right|^{2}}+1+\cos (2 \tau)\left(-4 \frac{a^{2}}{\left|\varphi_{w}\right|^{2}}+1\right)\right) .
\end{aligned}
$$

Motions satisfying $\left|\varphi_{w}\right|<a$ do not depend on the time delay $\tau$.

An additional change of variables is needed to reduce the system into an autonomous one. Considering the following new variables

$$
\hat{\varphi}_{u}=\varphi_{u} \mathrm{e}^{-j \epsilon \sigma t-j \phi}, \hat{\varphi}_{w}=\varphi_{w} \mathrm{e}^{-j \epsilon \sigma t-j \phi}
$$

Eqs. (15-16) are reduced to the autonomous form

$$
\begin{aligned}
\dot{\varphi}_{u}+j \epsilon \sigma \varphi_{u}+j \epsilon \frac{\left(\varphi_{u}-\varphi_{w}\right)}{2(1+\epsilon)}+\epsilon \mu \frac{\left(\varphi_{u}+\epsilon \varphi_{w}\right)}{1+\epsilon} & =\frac{1}{2} \epsilon A,(25) \\
\dot{\varphi}_{w}+j \epsilon \sigma \varphi_{w}+j \frac{\left(\varphi_{w}-\varphi_{u}\right)}{2(1+\epsilon)}+\epsilon \mu \frac{\left(\varphi_{u}+\epsilon \varphi_{w}\right)}{1+\epsilon} & \\
+(1+\epsilon) \varphi_{w}\left|\varphi_{w}\right|\left(G_{1}^{R}\left(\tau,\left|\varphi_{w_{\tau}}\right|\right)+j G_{1}^{I}\left(\tau,\left|\varphi_{w_{\tau}}\right|\right)\right) & \\
-\frac{j 3}{8} \lambda(1+\epsilon) \varphi_{w}\left|\varphi_{w}\right|^{2} & =\frac{1}{2} \epsilon A .(26)
\end{aligned}
$$

To simplify the hat sign $\left(^{\wedge}\right)$ has been omitted.

When $\tau=0$, only Case 1 is valid and Eqs. (25-26) coincide with results given in [9].

\subsection{Periodic solutions}

The periodic solutions of Eqs. (7-8) correspond to the fixed points $\left(\varphi_{u 0}, \varphi_{w 0}\right)$ of Eqs. (25-26). They are defined as the roots of the system of algebraic equations by imposing $\dot{\varphi}_{u}=0$ and $\dot{\varphi}_{w}=0$ in Eqs. (25-26). This system takes the following form

$$
\begin{aligned}
\left(\frac{\epsilon \mu}{1+\epsilon}+j(\epsilon \sigma\right. & \left.\left.+\frac{\epsilon}{2(1+\epsilon)}\right)\right) \varphi_{u} \\
+\left(\frac{\epsilon^{2} \mu}{1+\epsilon}-\frac{j \epsilon}{2(1+\epsilon)}\right) \varphi_{w}-\frac{1}{2} \epsilon A & =0, \\
\varphi_{w} P\left(\left|\varphi_{w}\right|\right) & =c_{0}
\end{aligned}
$$

where

$$
P(Z)=b_{0}+b_{1} Z+(1+\epsilon) Z\left(G_{1}^{R}(\tau, Z)+j G_{1}^{I}(\tau, Z)\right)-\frac{3}{8} j \lambda(1+\epsilon) Z^{2}
$$

and $b_{0}, b_{1}$ and $c_{0}$ are complex (not given here). Taking the complex conjugate of Eq. (28) and multiplying the resulting two equations together, one obtains the following real algebraic equation in $Z=\left|\varphi_{w}\right|$,

$$
Z^{2}|P(Z)|^{2}=c_{0} \overline{c_{0}} .
$$

The real positive roots $Z_{0}$ are obtained by solving separately Eq. (30) under the constraint $Z<a$ and under the constraint $Z>a$ with Case 1 and Case 2. Under the constraint $Z<a$ and under the constraint $Z>a$ with Case 1, Eq. (30) is a polynomial in $Z=\left|\varphi_{w}\right|$ with real coefficients easy to solve. Under the constraint $Z>a$ with Case 2, Eq. (30) is solved using a Newton method.

Knowing $Z_{0}$, the fixed points of Eqs. (25-26) are then deduced as

$$
\varphi_{w 0}=\frac{c_{0}}{P\left(Z_{0}\right)}, \varphi_{u 0}=\frac{-\left(\frac{\epsilon^{2} \mu}{1+\epsilon}-\frac{j \epsilon}{2(1+\epsilon)}\right) \varphi_{w 0}+\frac{1}{2} \epsilon A}{\left(\frac{\epsilon \mu}{1+\epsilon}+j\left(\epsilon \sigma+\frac{\epsilon}{2(1+\epsilon)}\right)\right)} .
$$

\subsection{Stability analysis}

The stability analysis of a periodic response of Eqs. (7-8) can be explored by analyzing the stability of the associated fixed points $\left(\varphi_{u 0}, \varphi_{w 0}\right)$ of Eqs. (25-26).

Re-writing Eqs. (25-26) as

$$
\dot{\varphi}=\mathbf{A} \varphi+\mathbf{B}(\varphi, \bar{\varphi})
$$

where $\varphi=\left(\varphi_{u}, \varphi_{w}\right)^{T}$, introducing the linearized terms of $\mathbf{B}$ and its conjugate $\overline{\mathbf{B}}$ around $(\varphi, \bar{\varphi})=\left(\varphi_{0}, \overline{\varphi_{0}}\right)$ where $\varphi_{0}=$ $\left(\varphi_{u 0}, \varphi_{w 0}\right)$ as

$$
\begin{aligned}
\mathbf{B}\left(\varphi_{0}+\delta \varphi, \overline{\varphi_{0}}+\overline{\delta \varphi}\right) & \approx \partial_{\varphi} \mathbf{B}\left(\varphi_{0}, \overline{\varphi_{0}}\right) \delta \varphi+\partial_{\bar{\varphi}} \mathbf{B}\left(\varphi_{0}, \overline{\varphi_{0}}\right) \overline{\delta \varphi} \\
\overline{\mathbf{B}}\left(\varphi_{0}+\delta \varphi, \overline{\varphi_{0}}+\overline{\delta \varphi}\right) & \approx \partial_{\varphi} \overline{\mathbf{B}}\left(\varphi_{0}, \overline{\varphi_{0}}\right) \delta \varphi+\partial_{\bar{\varphi}} \overline{\mathbf{B}}\left(\varphi_{0}, \overline{\varphi_{0}}\right) \overline{\delta \varphi}
\end{aligned}
$$

and linearizing Eq. (32) (and its conjugate equation) at $(\varphi, \bar{\varphi})=\left(\varphi_{0}, \overline{\varphi_{0}}\right)$, we obtain the following close linear system

$$
\left(\begin{array}{c}
\dot{\delta \varphi} \\
\dot{\delta \varphi}
\end{array}\right)=\left(\begin{array}{cc}
\left.\mathbf{A}+\partial_{\varphi} \mathbf{B}\left(\varphi_{0}, \overline{\varphi_{0}}\right)\right) & \partial_{\bar{\varphi}} \mathbf{B}\left(\varphi_{0}, \overline{\varphi_{0}}\right) \\
\partial_{\varphi} \overline{\mathbf{B}}\left(\varphi_{0}, \overline{\varphi_{0}}\right) & \bar{A}+\partial_{\bar{\varphi}} \overline{\mathbf{B}}\left(\varphi_{0}, \overline{\varphi_{0}}\right)
\end{array}\right)\left(\frac{\delta \varphi}{\delta \varphi}\right) .
$$

This system of equations should be solved separately for the two domain: $\left|\varphi_{w 0}\right|<a$ and $\left|\varphi_{w 0}\right|>a$.

The eigenvalues of the associated matrix characterize the local stability property of the fixed point $\varphi_{0}$.

\section{Some numerical results}

To illustrate the method and to analyze the influence of the time delay, results obtained solving Eq. (30) are compared with results obtained solving numerically Eqs. (7-9) by using the delay differential equations solver DDE23 available in (C) Matlab (with the default values for the argument $o p$ tions). The initial condition in the delay interval $[-\tau, 0]$ is assumed constant equal to zero vector.

The system is defined with $\epsilon=0.01, \mu=0.1$ and $\lambda=$ $3 / 4$. Six configurations of the NES damping are considered with $a=0.8$ and $\lambda_{m}=0.2$ : configuration $1: \lambda_{M}=0.2$ (quadratic), configuration 2: $\lambda_{M}=1$ (piecewise-quadratic), 
configuration 3: $\lambda_{M}=1$ and $\tau=0.1$ (delay piecewisequadratic), configuration 4: $\lambda_{M}=1$ and $\tau=0.3$ (delay piecewise-quadratic), configuration 5: $\lambda_{M}=1$ and $\tau=0.5$ (delay piecewise-quadratic) and configuration 6: $\lambda_{M}=5$ (piecewise-quadratic).

The forced responses associated to the periodic excitation are defined with amplitude excitation $A=1$ and the frequency excitation $\sigma$ in the interval $[-2.5,2.5]$ are reported in Fig. 2 in terms of $\operatorname{Max}\left|x_{1}-x_{2}\right|$ versus $\sigma$.

Configurations 1 and 6 have been considered in [9], to illustrate that a NES with a properly tuned piecewisequadratic damping element (see configuration 6 in Fig. 2) allows complete elimination of undesirable periodic regimes (see configuration 1 in Fig. 2). A similar results is obtained using delay piecewise-quadratic damping with a smaller tuned piecewise- quadratic damping element (see configuration 5 in Fig. 2).

The time delay seems to have the same effect as to increase the damping $\lambda_{M}$.
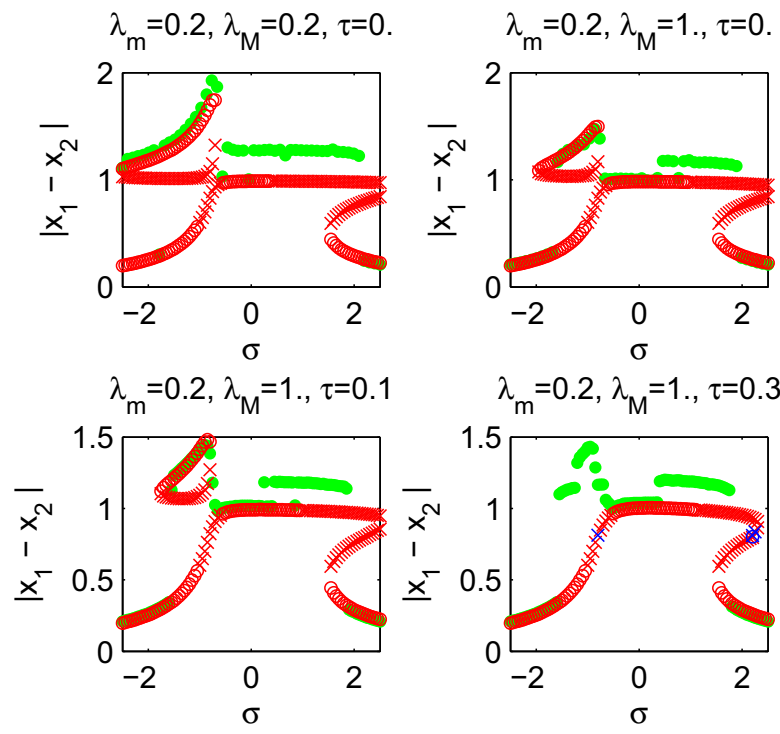

$\lambda_{\mathrm{m}}=0.2, \lambda_{\mathrm{M}}=1 ., \tau=0.3$

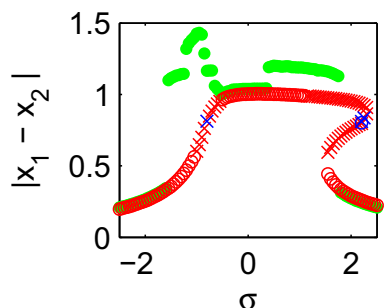

$\lambda_{\mathrm{m}}=0.2, \lambda_{\mathrm{M}}=1 ., \tau=0.5$ $\lambda_{\mathrm{m}}=0.2, \lambda_{\mathrm{M}}=5 ., \tau=0$.
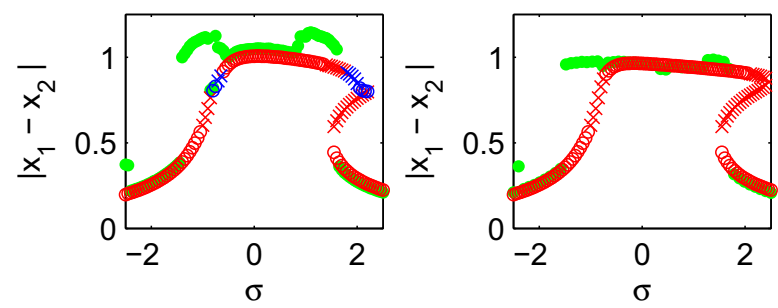

Fig. 2. Max $\left|x_{1}-x_{2}\right|$ versus $\sigma$ from Eq. (30) (blue and red markers) and solving numerically Eqs. (7-9) (green point markers) for the six configurations. Circle markers refer to stable solutions and cross markers refer to unstable solutions. Blue markers refers to Case 2.

\section{Conclusion}

The efficiency of a nonlinear attachment including nonlinear damping with time delay as an nonlinear absorber has been analyzed using the complexification method. Further works will be developed employing a nonlinear time transformation method. This method is able to find accurate analytical solution at all powers of $\epsilon$. The method was applied in $[10,11]$ to obtain limit cycles arisen from Hopf bifurcation.

\section{References}

1. R. Bellet, B. Cochelin, P. Herzog, P.-O. Mattei, Experimental study of targeted energy transfer from an acoustic system to a nonlinear membrane absorber, Journal of Sound and Vibration 329 (2010) 27682791.

2. R. Mariani, S. Bellizzi, B. Cochelin, P. Herzog, P.-O. Mattei, Toward an adjustable nonlinear low frequency acoustic absorber, Journal of Sound and Vibration 330 (2011) 5245-5258.

3. O. Gendelman, L. Manevitch, A. Vakakis, R. Mloskey, Energy pumping in nonlinear mechanical oscillators: Part I - Dynamics of the underlying hamiltonian systems, Journal of Applied Mechanics 68 (2001) 34-41.

4. A. Vakakis, O. Gendelman, Energy pumping in nonlinear mechanical oscillators: Part II - Resonance capture, Journal of Applied Mechanics 68 (2001) 42-48.

5. E. Gourdon, N. Alexander, C. Taylor, C. Lamarque, $\mathrm{S}$. Pernot, Non- linear energy pumping under transient forcing with strongly nonlinear coupling: Theoretical and experimental results, Journal of Sound and Vibration 300 (2007) 522-551.

6. A. Vakakis, L. Manevitch, O. Gendelman, L. Bergman, Dynamics of linear discrete systems connected to local, essentially non-linear attach- ments, Journal of Sound and Vibration 264 (2003) 559-577.

7. O. Gendelman, Y. Starosvetsky, M. Feldman, Attractors of harmonically forced linear oscillator with attached nonlinear energy sink i:Description of response regimes, Nonlinear Dynamics 51 (2008) 31-46.

8. Y. Starosvetsky, O. V. Gendelman, Attractors of harmonically forced linear oscillator with attached nonlinear energy sink. ii: Optimization of a nonlinear vibration absorber, Nonlinear Dynamics 51 (2008) 4757.

9. Y. Starosvetsky, O. Gendelman, Vibration absorption in systems with a nonlinear energy sink: Nonlinear damping, Journal of Sound and Vibration 324 (2009) 916-939.

10. J. Xu, K. W. Chung, C. L. Chan, An efficient method for studying weak resonant double Hopf bifurcation in nonlinear systems with delayed feedbacks, SIAM Journal of Applied Dynamical Systems, 6(1), 29-60, 2007.

11. K. W. Chung, Z. H. Liu. Nonlinear analysis of chatter vibration in a cylindrical transverse grinding process with two time delays using a nonlinear time transformation method, Nonlinear Dynamics, 66, 441-456, 2011 . 